\title{
Author Correction: A systems biology approach uncovers cell-specific gene regulatory effects of genetic associations in multiple sclerosis
}

International Multiple Sclerosis Genetics Consortium

Correction to: Nature Communications https://doi.org/10.1038/s41467-019-09773-y, published online 20 May 2019.

The original version of this Article contained an error in the spelling of the author Nikolaos A. Patsopoulos, which was incorrectly given as Niklaos A. Patsopoulos, and author Efthimios Dardiotis, which was incorrectly given as Dardiotis Efthimios. This has now been corrected in both the PDF and HTML versions of the Article.

Published online: 01 July 2019

\section{International Multiple Sclerosis Genetics Consortium}

Lohith Madireddy', Nikolaos A. Patsopoulos ${ }^{2,3}$, Chris Cotsapas4,5, Steffan D. Bos 6,7, Ashley Beecham ${ }^{8}$, Jacob McCauley ${ }^{8,9}$, Kicheol Kim¹, Xiaoming Jia', Adam Santaniello1, Stacy J. Caillier ${ }^{1}$, Till F.M. Andlauer ${ }^{10,11}$, Lisa F. Barcellos ${ }^{12}$, Tone Berge 7,13, Luisa Bernardinellii ${ }^{14}$, Filippo Martinelli-Boneschi ${ }^{15,16}$, David R. Booth ${ }^{17}$, Farren Briggs ${ }^{18}$, Elisabeth G. Celius ${ }^{7,19}$, Manuel Comabella ${ }^{20}$, Giancarlo Comi ${ }^{21}$, Bruce A.C. Cree , Sandra D'Alfonso ${ }^{22}$, Katrina Dedham ${ }^{23}$, Pierre Duquette ${ }^{24}$, Efthimios Dardiotis ${ }^{25}$, Federica Esposito ${ }^{21}$, Bertrand Fontaine ${ }^{26,27}$, Christiane Gasperi ${ }^{10}$, An Goris ${ }^{28}$, Bénédicte Dubois ${ }^{28}$, Pierre-Antoine Gourraud ${ }^{29,30}$, Georgios Hadjigeorgiou ${ }^{31}$, Jonathan Haines ${ }^{18}$, Clive Hawkins ${ }^{32,37}$, Bernhard Hemmer ${ }^{10}$, Rogier Hintzen ${ }^{33,34}$, Dana Horakova ${ }^{35}$, Noriko Isobe ${ }^{36}$, Seema Kalra ${ }^{32,37}$, Jun-ichi Kira ${ }^{36}$, Michael Khalil ${ }^{38}$, Ingrid Kockum ${ }^{39}$, Christina M. Lill ${ }^{40,41}$, Matthew R. Lincoln ${ }^{4}$, Felix Luessi ${ }^{40}$, Roland Martin ${ }^{42}$, Annette Oturai ${ }^{43}$, Aarno Palotie ${ }^{44,45}$, Margaret A. Pericak-Vance ${ }^{8,9}$, Roland Henry', Janna Saarela44, Adrian Ivinson ${ }^{46}$, Tomas Olsson ${ }^{39}$, Bruce V. Taylor ${ }^{47}$, Graeme J. Stewart ${ }^{17}$, Hanne F. Harbo ${ }^{6,7}$, Alastair Compston ${ }^{48}$, Stephen L. Hauser ${ }^{1}$, David A. Hafler ${ }^{4}$, Frauke Zipp ${ }^{40}$, Philip De Jager ${ }^{3,49}$, Stephen Sawcer ${ }^{48}$, Jorge R. Oksenberg ${ }^{1}$ \& Sergio E. Baranzini (10) 1,50

${ }^{1}$ Weill Institute for Neurosciences, Department of Neurology, University of California San Francisco, San Francisco, CA 94158, USA. ${ }^{2}$ Systems Biology and Computer Science Program, Ann Romney Center for Neurological Diseases, Department of Neurology, and Division of Genetics, Department of Medicine, Brigham \& Women's Hospital, Harvard Medical School, Boston, MA 02115, USA. ${ }^{3}$ Broad Institute of Harvard University and Massachusetts Institute of Technology, Cambridge, MA 02142, USA. ${ }^{4}$ Departments of Neurology, Yale School of Medicine, 300 George St, New Haven, CT 06511, USA. ${ }^{5}$ Department of Genetics, Yale School of Medicine, 300 George St, New Haven, CT 06511, USA. ${ }^{6}$ Institute of Clinical 
Medicine, University of Oslo, Oslo 0318, Norway. ${ }^{7}$ Department of Neurology, Oslo University Hospital, Oslo 0424, Norway. ${ }^{8}$ John P. Hussman Institute for Human Genomics, University of Miami, Miller School of Medicine, Miami, FL 33136, USA. ${ }^{9}$ Dr. John T. Macdonald Foundation Department of Human Genetics, University of Miami, Miller School of Medicine, Miami, FL 33136, USA. ${ }^{10}$ Department of Neurology, Klinikum rechts der Isar, School of Medicine, Technical University of Munich, 81675 Munich, Germany. ${ }^{11}$ Munich Cluster for Systems Neurology (SyNergy), 81377 Munich, Germany. ${ }^{12}$ Division of Epidemiology, School of Public HealthUniversity of California, 324 Stanley Hall, MC\#3220, Berkeley, CA 94720, USA. ${ }^{13}$ Department of Mechanical, Electronics and Chemical Engineering, Oslo Metropolitan University, Oslo 0167, Norway. ${ }^{14}$ Section of Biostatistics, Neurophyisiology and Psychiatry, Unit of medical and genomic statistics, Universita of Pavia, Pavia 27100, Italy. ${ }^{15}$ Department of Biomedical Sciences for Health, University of Milan, Milan 20133, Italy. ${ }^{16}$ MS Research Unit and Department of Neurology, IRCCS Policlinico San Donato, San Donato Milanese, Milan 20097, Italy. ${ }^{17}$ Faculty of Medicine, Westmead Clinical School, The Westmead Institute for Medical Research, Sydney, NSW 2145, Australia. ${ }^{18}$ Department of Quantitative and Population Health Sciences, School of Medicine, Case Western Reserve University, Cleveland, $\mathrm{OH} 44106, \mathrm{USA} .{ }^{19}$ Institute of Health and Society, University of Oslo, Oslo 0318, Norway. ${ }^{20}$ Servei de Neurologia-Neuroimmunologia. Centre d'Esclerosi Múltiple de Catalunya (Cemcat), Institut de Recerca Vall d'Hebron (VHIR), Hospital Universitari Vall d'Hebron, Universitat Autònoma de Barcelona, Barcelona 08035, Spain. ${ }^{21}$ Department of Neurology, San Raffaele Scientific Institute, Milan 20132, Italy. ${ }^{22}$ Department of Health Sciences, UPO University, Novara 28100, Italy. ${ }^{23}$ Department of Clinical Neurosciences. Neurology Unit, University of Cambridge, Cambridge CB2 1QW, UK. ${ }^{24}$ Faculté de médecine, MS Clinic Centre Hospitalier de I', Université de Montréal. Université de Montréal Montreal, Montreal QC H3A 1G1, Canada. ${ }^{25}$ Department of Neurology, Laboratory of Neurogenetics, University of Thessaly, University Hospital of Larissa, Larissa 41223, Greece. ${ }^{26}$ Department of Neurology, University Hopital Pitié-Salpêtrière, Paris 75013, France. ${ }^{27}$ UMR 1127, Sorbonne-Université, INSERM, University Hopital Pitié-Salpêtrière, Paris 75013, France. ${ }^{28} \mathrm{KU}$ Leuven Department of Neurosciences, Laboratory for Neuroimmunology, Leuven 3000, Belgium. ${ }^{29}$ Université de Nantes, INSERM, Centre de Recherche en Transplantation et Immunologie, UMR 1064, ATIP-Avenir, Equipe 5, Nantes F-44093, France. ${ }^{30} \mathrm{CHU}$ de Nantes, INSERM, CIC 1413, Pôle Hospitalo-Universitaire 11: Santé Publique, Clinique des données, Nantes F44093, France. ${ }^{31}$ Department of Neurology, Medical School, University of Cyprus, Nicosia 587G+X2, Cyprus. ${ }^{32}$ Institute for Science \& Technology in Medicine, Keele University, Keele ST5 5GB, UK. ${ }^{33}$ Department of Neurology, Erasmus MC Dr Molewaterplein 40, Rotterdam 3015 GD, The Netherlands. ${ }^{34}$ Department of Immunology, Erasmus MC, Rotterdam 3015 GD, The Netherlands. ${ }^{35}$ First Faculty of Medicine, Department of Neurology and Center of Clinical Neuroscience, Charles University and General University Hospital, Prague 3CFG+RJ, Czech Republic.

${ }^{36}$ Department of Neurology, Kyushu University, Kyushu 812-0053, Japan. ${ }^{37}$ Royal Stoke MS Centre of Excellence, University Hospital North Midlands, Stoke-on-Trent, ST4 6QG, UK. ${ }^{38}$ Department of Neurology, Medical University of Graz, Graz A-8036, Austria. ${ }^{39}$ Department of Clinical Neuroscience and Center for Molecular Medicine, Karolinska Institutet, Stockholm 17176, Sweden. ${ }^{40}$ Department of Neurology, University Medical Center of the Johannes Gutenberg University Mainz, Mainz 55131, Germany. ${ }^{41}$ Genetic and Molecular Epidemiology Group, Lübeck Interdisciplinary Platform for Genome Analytics, Institutes of Neurogenetics and Cardiogenetics, University of Lübeck, Lübeck 23562, Germany.

${ }^{42}$ Neuroimmunology and MS Research (nims), Department of Neurology, University Zurich, Zürich 8006, Switzerland. ${ }^{43}$ Department of Neurology, section 2082, Rigshospitalet, Danish Multiple Sclerosis Center, University of Copenhagen, Copenhagen 2100, Denmark. ${ }^{44}$ Institute for Molecular Medicine Finland (FIMM), University of Helsinki, Helsinki FIN-00014, Finland. ${ }^{45}$ Harvard University, Center for Human Genetic Research, Boston, MA 02115, USA. ${ }^{46}$ UK Dementia Research Institute at University College London, London WC1E6BT, UK. ${ }^{47}$ Menzies Institute for Medical Research, University of Tasmania, Hobart, TAS 7000, Australia. ${ }^{48}$ Department of Clinical Neurosciences, Cambridge Biomedical Campus, University of Cambridge, Cambridge CB2 OQQ, UK. ${ }^{49}$ Department of Neurology, Center for Translational and Computational Neuroimmunology and Multiple Sclerosis Center, Columbia University Medical Center, New York, NY 10032, USA. ${ }^{50}$ Bakar Institute for Computational Health Science. University of California San Francisco, San Francisco, CA 94158, USA

Open Access This article is licensed under a Creative Commons Attribution 4.0 International License, which permits use, sharing, adaptation, distribution and

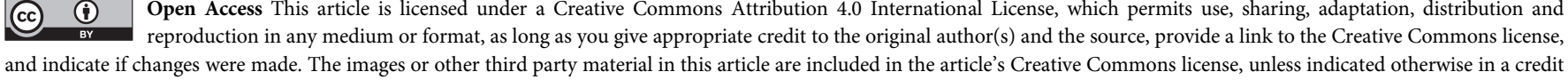
line to the material. If material is not included in the article's Creative Commons license and your intended use is not permitted by statutory regulation or exceeds the permitted use, you will need to obtain permission directly from the copyright holder. To view a copy of this license, visit http://creativecommons.org/licenses/by/4.0/.

(c) The Author(s) 2019 